\title{
Effects of inflation on the subjective value of delayed and probabilistic rewards
}

\author{
PAWEL OSTASZEWSKI \\ University of Warsaw, Warsaw, Poland \\ and \\ LEONARD GREEN and JOEL MYERSON \\ Washington University, St. Louis, Missouri
}

\begin{abstract}
In the years prior to 1994 , there were very high rates of inflation in Poland, and the zloty depreciated relative to the U.S. dollar. However, the new zloty, introduced in 1995, was associated with greatly decreased rates of inflation and provided a more stable currency. We report a series of three experiments that take advantage of these changes to examine the effects of inflation on the subjective value of delayed and probabilistic rewards. Subjects were Polish citizens familiar with both zlotys and dollars. The first two experiments, conducted in 1994, used dollars and old zlotys, and the third experiment, conducted in 1996, used dollars and new zlotys. In all three experiments, the dollar and zloty rewards were of equivalent worth, according to the then current exchange rates. In Experiment 1, subjects chose between immediate and delayed rewards and, in Experiment 2, chose between certain and probabilistic rewards. The subjective value of a delayed reward was greater when its amount was specified in dollars than when it was specified in old zlotys. In contrast, the currency in which a reward was specified had no effect on the subjective value of probabilistic rewards. The results of these two experiments suggest a selective effect of inflation on decisions involving delayed rewards. This was verified in the third experiment, in which, using new zlotys, no differences in discounting were observed between the two currencies with either probabilistic or delayed rewards. Importantly, in all three experiments, the discounting of both delayed and probabilistic rewards was well described by the same simple mathematical model, suggesting that similar decision-making processes underlie both phenomena. However, the present results argue against a single-process theory in which the discounting of probabilistic rewards is derived from the discounting of delayed rewards.
\end{abstract}

Recent years have witnessed the growth and development of a new field of research known as behavioral economics. A defining property of behavioral economics is the use of experimental psychological methods to examine economic issues. Both disciplines have profited, theoretically as well as empirically, from research in this area. Behavioral economics provides an experimental laboratory in which to evaluate economic models and examine the psychological bases for economic phenomena (e.g., Green \& Kagel, 1987, 1990; Kagel, Battalio, \& Green, 1995; Lea, Tarpy, \& Webley, 1987). As behavioral economics has developed, it has lead to the incorporation of economic concepts into psychological theory and enriched our thinking about choice and decision making

This research was supported in part by Grants BST 505/40 and 1 H01F 01510 from the Scientific Research Committee (KBN), Poland, and by Grant R01 MH55308 from the National Institute of Mental Health. Pawel Ostaszewski was supported as a Visiting Scholar at Washington University by a fellowship from the Kosciuszko Foundation. Portions of this research were presented at the 1996 meeting of the Psychonomic Society in Chicago. Address correspondence to P. Ostaszewski, Faculty of Psychology, Warsaw University, Stawki 5/7, 00183 Warsaw, Poland, or L. Green, Department of Psychology, Washington University, Campus Box 1125, St. Louis, MO 63130 (e-mail: lgreen@ artsci.wustl.edu). (e.g., Allison, 1983; Hursh, 1980, 1984; Rachlin, 1989; Staddon, 1980).

To date, most behavioral economic research has examined microeconomic issues, focusing on the determinants of behavior in individuals. In contrast, little attention has been paid to macroeconomic issues involving economic aggregates such as level of unemployment, national output of goods and services, and rates of inflation. The present investigation is unique in that it takes advantage of events at the macroeconomic level, specifically differential changes in rates of inflation in two different currencies, and examines the consequences of these macroeconomic changes for individual choice and decision making. The effects on individual choice and decision making, in turn, are of interest in part because of their implications for the behavior of economic aggregates.

\section{Discounting of Delayed and Probabilistic Outcomes}

A fundamental psychological and microeconomic phenomenon is the fact that individuals discount the value of delayed and probabilistic rewards. For example, most people would choose an immediate reward over a delayed reward of the same amount, presumably because they discount the value of the delayed reward. Similarly, people would choose a certain reward over a reward of the 
same amount whose receipt is uncertain, presumably because they discount the value of the uncertain reward.

Previous research on temporal discounting (i.e., the decrease in the present, subjective value of a reward as the delay until its receipt increases) has shown that choices are well described by a hyperbola-like function:

$$
V=A /(1+k D)^{s}
$$

where $V$ is the present subjective value of a reward of amount $A$ to be received after a delay, $D$ (Green, Fry, \& Myerson, 1994). The parameter $k$ reflects the rate at which subjective value decreases, and the parameter $s$ reflects the nonlinear scaling of amount and/or time. When $s$ equals 1.0 , Equation 1 is the simple hyperbola that has been used to describe temporal discounting by both animal and human subjects deciding between hypothetical or actual rewards (e.g., Kirby, 1996; Kirby \& Maraković, 1995; Mazur, 1987; Rachlin, Raineri, \& Cross, 1991; Rodriguez \& Logue, 1988). However, compared with the simple hyperbola, Equation 1 is more general and better describes discounting by individuals (Myerson \& Green, 1995).

Discounting of probabilistic rewards involves choices between outcomes that differ in certainty but in which rewards, if received at all, are received immediately. Rachlin et al. (1991) showed that the discounting of probabilistic rewards (i.e., the decrease in the subjective value of a reward as the odds against its receipt increase) is described by an equation of similar form:

$$
V=A /(1+h \Theta)
$$

where $\Theta$ represents the odds against receipt of a reward [i.e., $\Theta=(1-p) / p$, where $p$ is the probability of receipt] and $h$ is a parameter (analogous to $k$ in Equation 1) that reflects the rate of decrease in subjective value. Equation 2 may be made formally equivalent to Equation 1 by raising the denominator to a power, $s$ :

$$
V=A /(1+h \Theta)^{s} \text {. }
$$

Equation 3 has not been evaluated previously. One goal of the present research was to evaluate Equation 3 in order to determine whether a function of the same form as Equation 1, the temporal discounting function, could accurately describe the discounting of probabilistic rewards. This question is important in part because of its implications for theories that assume that similar or even identical processes are involved in decisions regarding delayed and probabilistic rewards. For example, Rachlin and his colleagues (Rachlin, Logue, Gibbon, \& Frankel, 1986; Rachlin et al., 1991) have proposed a single-process discounting model that explains the discounting of probabilistic rewards in terms of an underlying temporal discounting process. Myerson and Green (1995; Green \& Myerson, 1996) have also proposed a single-process account, but have suggested that discounting of probabilistic rewards might underlie temporal discounting. In addition, Prelec and Loewenstein (1991) have suggested that decision making regarding rewards was similar to other psychophysical judgments and that, because of this similarity, similar functions described the discounting of both delayed and probabilistic rewards.

Although they differ in specifics, all of these theorists postulate a fundamental similarity between the discounting of both delayed and probabilistic rewards and predict that the same form of mathematical function will describe both phenomena. Discounting of delayed rewards is best described by a function of the form of Equation 1 (Green et al., 1994; Myerson \& Green, 1995), but it is unknown whether a function of the same form (i.e., Equation 3) also describes discounting of probabilistic rewards. Although it was clear that the discounting functions were qualitatively similar in both cases, one goal of the present study was to determine whether they were truly isomorphic.

\section{Inflation and Discounting}

One might think that inflation could completely account for the discounting of delayed rewards. After all, people may value delayed rewards less than rewards of the same amount received sooner because inflation would reduce the relative purchasing power of the more delayed rewards. However, animals show temporal discounting of food rewards (e.g., Green, Fisher, Perlow, \& Sherman, 1981; Mazur, 1987), and the rate at which people in the U.S. discount future rewards greatly exceeds what would be predicted from actual inflation rates (e.g., Hausman, 1979; Thaler, 1981). Therefore, inflation clearly is not a sufficient explanation for all discounting of delayed rewards. However, expectations regarding future inflation might still explain some cases of discounting by humans or play a role in modulating the degree to which they discount the value of delayed rewards.

The hypothesis that inflation modulates discounting is difficult to test because researchers cannot experimentally manipulate inflation. In spite of this constraint, macroeconomic events may afford opportunities for quasiexperiments. For many years, the rate of inflation in Poland in terms of the buying power of zlotys was so high that many people kept their savings in dollars. For example, between 1989 and 1993, the rate of inflation (as measured by annual percent change in consumer prices) averaged over 100\% (International Monetary Fund, 1996). Importantly, there were special stores (called Pewex stores) where the goods were priced in dollars and could not be purchased with zlotys; as a result, many people in Poland are used to trading in both currencies and are familiar with their different rates of depreciation. Thus, the influence of inflation may be revealed by comparing the way in which they discount dollar and zloty rewards and by comparing observed discounting with the decrease in purchasing power predicted by actual rates of inflation.

The hypothesized modulatory effect of inflation on discounting may be specific to decisions involving future rewards. That is, inflation may decrease the purchasing 
power of delayed rewards relative to immediate rewards, but not differentially affect the purchasing power of certain and probabilistic rewards. If so, one would expect that, because of the differential rates of inflation in dollars and zlotys, zloty rewards would be discounted more steeply than dollars when rewards were delayed but that zloty and dollar rewards would be discounted at equivalent rates when rewards were uncertain. Such selective modulation would be contrary to the predictions of singleprocess theories of discounting, at least as currently proposed. After all, if both probability of reward and delay to reward are converted to the same metric, be it one based on risk (Myerson \& Green, 1995) or one based on delay (Rachlin et al., 1991), then whatever effects of inflation are observed with probabilistic rewards should also be observed with delayed rewards (and vice versa).

Accordingly, the present study tested whether inflation influenced the discounting of monetary rewards and whether this influence was general (affecting both delayed and probabilistic rewards) or was specific to delayed rewards. In Experiments 1 and 2 the discounting of zloty and dollar rewards at a time (1994) immediately following a period when these two currencies differed markedly in inflation rates was compared. In Experiment 3, conducted in 1996, a year after a new Polish currency was introduced, the effect of currency differences when the differences in inflation rates were much lower was examined. Furthermore, the present study provided the opportunity to compare the observed discounting of delayed rewards with that predicted on the basis of actual inflation rates. Such a comparison should help in distinguishing a modulatory effect of inflation from discounting that corresponds to realistic expectations as to future inflation rates. Finally, taken together, the three experiments also afforded the opportunity to assess whether the functions that described discounting of delayed and probabilistic rewards had the same mathematical form, as predicted by theories that assume that either the same or at least similar processes underlie discounting in both cases.

\section{EXPERIMENT 1}

In the first experiment, we examined whether the temporal discounting of rewards was affected by the currency in which the rewards were specified. This experiment was conducted in 1994, prior to the Polish monetary reform, and amounts were specified in the old zlotys (Zl) and in U.S. dollars (\$). For each currency, two delayed reward amounts were studied. These amounts were selected so that both the two small rewards ( $\$ 100$ and 2 million $\mathrm{Zl}$ ) and the two large rewards $(\$ 1,000$ and 20 million $\mathrm{Zl})$ were equivalent in worth according to the exchange rate at the time of the experiment. All previous studies that had examined the form of the relation between delay and the subjective value of monetary rewards had used amounts specified in dollars; the present experiment was the first to directly compare discounting in two different currencies.

\begin{abstract}
Method
Subjects. Twenty-five students at the University of Warsaw participated.

Procedure. The procedure was a computerized version of that used in Rachlin et al, (1991) and Green et al. (1994). Subjects made a series of choices between hypothetical amounts of money, one available after a delay and one available immediately (e.g., between " $\$ 100$ in one year" and " $\$ 10$ now"). The amount of the delayed reward was displayed on the right side of the monitor screen together with information about how long the delay would be (e.g., " $\$ 100$ in one year"); the amount of the immediate reward (e.g., "\$10 now") was displayed on the left side of the screen. Subjects indicated their choices by pressing one of two keys on the computer keyboard: either " 1 " for the immediate amount or " 0 " for the delayed amount.

The amounts of the delayed rewards were 100 and 1,000 U.S. dollars and 2 million and 20 million Polish zlotys. Each of these four amounts was presented at each of seven delays: 3 months, 6 months, 1 year, 3 years, 5 years, 10 years, and 20 years. There were 25 immediate amounts ranging from $1 \%$ to $99 \%$ of the delayed amount. At each delayed amount, immediate reward amounts were presented in both ascending and descending order. When the order was ascending, the immediate reward was increased until the subject indicated that the immediate reward was preferred to the delayed reward; when the order was descending, the immediate reward was decreased until the delayed reward was preferred.

This procedure was followed at each of the seven delays for all four delayed amounts. For half of the subjects, the order of the delayed amounts was 2 million Zl, 20 million $\mathrm{Zl}, \$ 100$, and $\$ 1,000$. For the other half of the subjects, the reverse order was followed. The order of delayed amounts was crossed with the order of the immediate amounts; half of the subjects were studied with ascending amounts preceding descending amounts; the other half were studied with descending preceding ascending. The delays were always studied in increasing order (i.e., 3 months to 20 years). For each delayed amount at each delay, the subjectively equivalent immediate amount was calculated for each subject as the average of the amount at which preference switched from the delayed to the immediate reward on the ascending series and from the immediate to the delayed reward on the descending series.
\end{abstract}

\section{Results and Discussion}

Figure 1 shows the amount of the immediate reward judged equal in subjective value to the delayed reward, expressed as a proportion of the amount of the delayed reward. The subjective value of the delayed reward decreased systematically as a function of delay for both amounts in each currency. The curved lines represent Equation 1 fit to data from a delayed dollar reward and the corresponding delayed zloty reward, simultaneously using a single exponent $(s)$ but a separate discounting parameter $(k)$ for each currency in order to compare the discounting rates for rewards specified in dollars and zlotys. ${ }^{1}$

The upper left panel of Figure 1 shows the group median data and best-fitting function for the smaller amounts in the two currencies (i.e., $\$ 100$ and 2 million $\mathrm{Zl}$ ); the upper right panel shows the data and best-fitting function for the larger amounts (i.e., $\$ 1,000$ and 20 million $\mathrm{Zl}$ ). Equation 1 accounted for $96.2 \%$ of the variance in the data for the smaller delayed amounts and $95.9 \%$ of the variance for the larger amounts. These results are consistent with previous reports that temporal discounting is well described by a hyperbola-like function.

Simple $t$ tests with $n-p$ degrees of freedom, where $n$ is the number of data points and $p$ is the number of estimated parameters (Gallant, 1987), were used to assess whether the exponent $s$ in Equation 1 differed significantly 


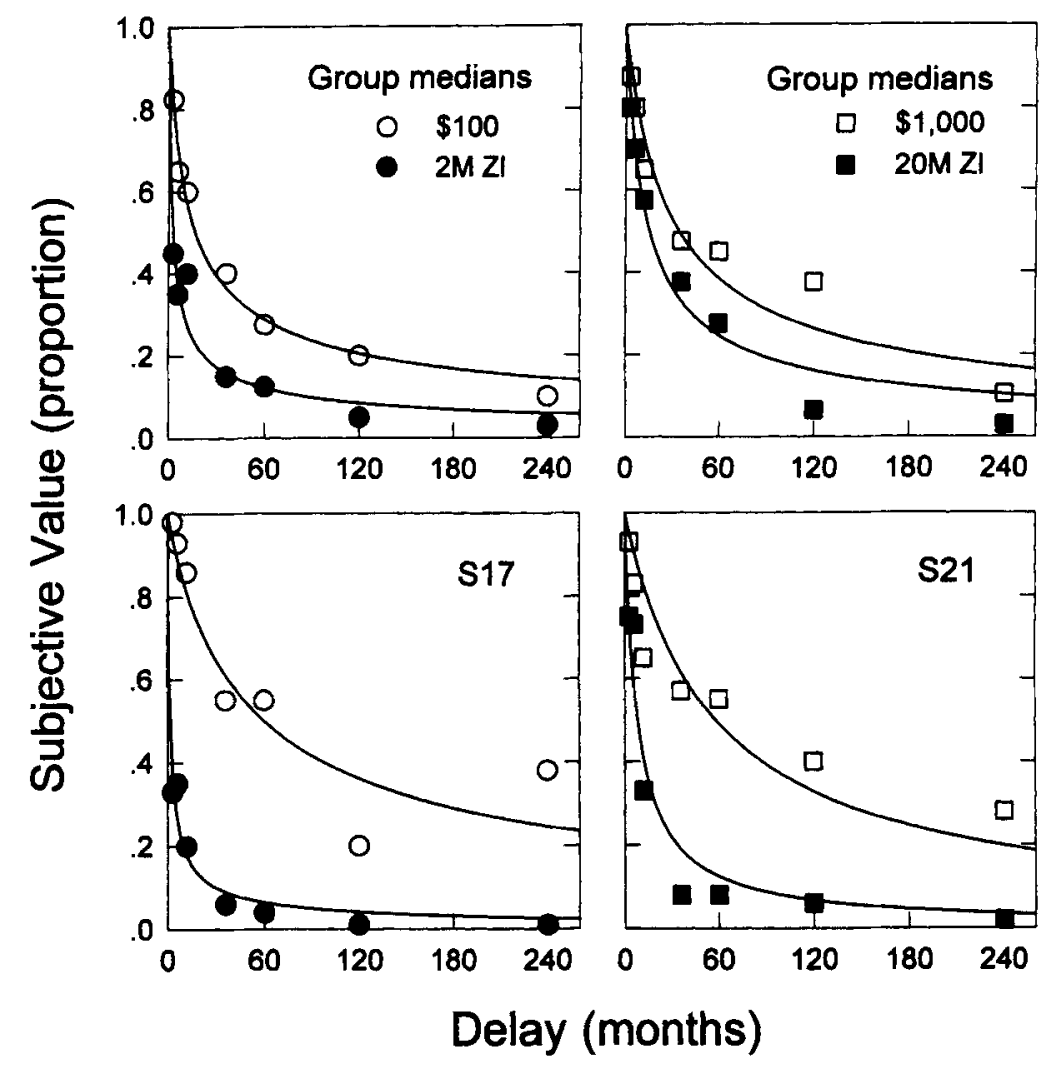

Figure 1. Subjective value of a reward as a function of the delay until its receipt. Data are the amount of immediate reward equal in subjective value to the delayed reward (Experiment 1). The subjective value of a reward is represented as a proportion of its nominal amount. Curves represent the best-fitting hyperbola-like discounting functions (Equation 1). Top panels show group medians, and bottom panels show data from representative individuals.

from 1.0, the value predicted under the null hypothesis. The $t$ value was calculated as the ratio of 1.0 minus the estimate of $s$ to the standard error of the estimate. The exponent was reliably less than 1.0 for both the small $(s=0.52)$ and large $(s=0.67)$ reward amounts $[t(11)=$ 6.39 and 2.27 , respectively, both $p s<.05]$. This result is consistent with our recent finding that raising the denominator of the discounting function to a power less than 1.0 provides a more accurate description than that provided by a simple hyperbola (Green et al. 1994; Myerson \& Green, 1995).

Equation 1 was also fit to the data from each individual subject separately. Data and best-fitting functions for the subjects with the median $R^{2} \mathrm{~s}$ for the smaller and larger amounts are presented in the two bottom panels. For these two subjects, Equation 1 accounted for $95.5 \%$ and $92.2 \%$ of the variance, respectively.

The results shown in Figure 1 are consistent with the hypothesis that delayed rewards in Polish zlotys are discounted more steeply than delayed rewards in U.S. dollars. The statistical procedure used to evaluate whether the rates of discounting differed between the two currencies was similar to that used to evaluate the exponent parameter. That is, evaluation was based on the value of $t$ (with $n-p$ degrees of freedom) calculated as the estimate of the difference between the two $k$ parameters divided by the standard error of the estimate. For the smaller amounts ( $\$ 100$ and 2 million $\mathrm{Zl}$ ), the discounting parameter $(k)$ was 0.165 for the dollar reward and 0.928 for the zloty reward $[t(11)=2.41, p<.05]$. For the larger amounts ( $\$ 1,000$ and 20 million $\mathrm{Zl}$ ), $k$ was 0.052 for the dollar reward and 0.116 for the zloty reward. Again, the zloty rewards were discounted more steeply, as indicated by a significantly larger $k$ parameter $[t(11)=2.04, p<$ $.05]$. These group results reflect the individual data: On average, $72 \%$ of the subjects discounted the zloty rewards more steeply than the corresponding dollar rewards.

Thus, for both the smaller and larger pairs of amounts in the two currencies, rewards of equivalent worth according to current exchange rates were not judged to be of equivalent subjective value. Rather, the subjective value of zloty rewards was discounted more steeply, so much 
so, in fact, that the subjective value of a zloty reward was less than that of the comparable dollar reward even when the delay to their receipt was as brief as 3 months.

Finally, these results replicated and extended our previous finding that the rate of temporal discounting varies inversely with amount (Green et al., 1994; Green, Myerson, \& McFadden, 1997; Myerson \& Green, 1995). When Equation 1 was fit to data from the smaller and larger rewards in the same currency, using a single exponent but a separate discounting parameter for each amount, the $k$ parameter was, as expected, significantly larger (indicating steeper discounting) for the smaller amount in both cases $[t(11)=2.28$ and 2.05 for the dollar and zloty rewards, respectively, both $p \mathrm{~s}<.05]$.

Is it possible that the observed discounting can be completely explained in terms of inflation's effect on the value of delayed rewards? To answer this question, one can compare the present results with what would be predicted on the basis of actual inflation rates. Taking inflation's effect on purchasing power into account leads to an exponential decrease in actual value over time. The upper curve (long dashes) in Figure 2 shows the predicted decline in value based on the inflation rate for the zloty in the immediately preceding year $(35.3 \%$ according to the Central Bureau for Statistics in Poland). Because inflation had been even higher in previous years and subjects might have used an average over some unknown number of years, the best-fitting exponential function is also shown (see the lower curve, short dashes). It can be seen that neither curve provides a good fit to the data.

It can also be seen that discounting based purely on inflation, regardless of the assumed rate, always predicts that large and small amounts will show equivalent pro-

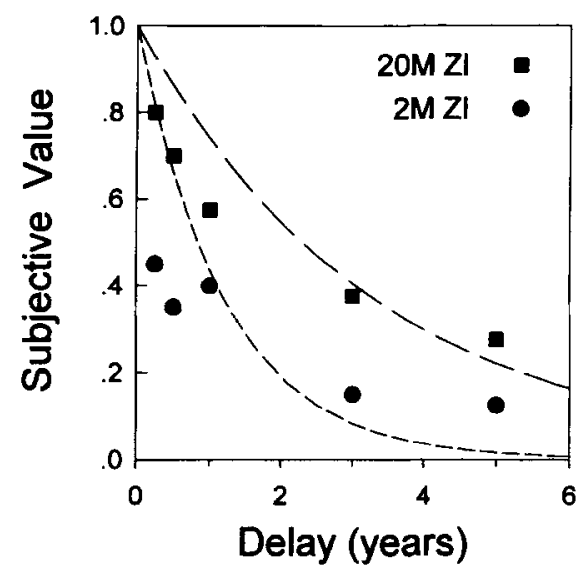

Figure 2. Effect of inflation on the predicted value of delayed zloty rewards. The two curves show decreases in actual value predicted by inflation on the basis of the rate observed in 1993 (upper curve, long dashes), the year prior to the experiment, and at the rate estimated by the best-fitting exponential function (lower curve, short dashes). The data points are taken from Figure 1 and represent the subjects' estimates of the subjective value of 2- and 20-million zloty rewards. Only delays out to 5 years are shown, since the curves approach zero at long delays. portional decreases in subjective value. Since the smaller amount was discounted more steeply than the larger amount in both currencies, this prediction is clearly not consistent with the data. Thus, although subjects' experience with inflation may have influenced their decision making, they were clearly doing more in the present experiment than simply estimating inflation's effect on future value.

\section{EXPERIMENT 2}

Experiment 1 showed that the rate at which delayed rewards were discounted differed depending on the currency, and that the same hyperbola-like function (Equation 1) described temporal discounting in both currencies. Experiment 2 (which, like Experiment 1, was conducted in 1994) tested whether the currency in which amounts are specified also leads to differences in the rate at which probabilistic rewards are discounted. The larger issue here was whether differences in discounting between currencies were specific to decisions involving time and future rewards or whether they were general and occurred with other kinds of decisions involving monetary rewards as well. In addition, Experiment 2 examined whether the discounting of probabilistic rewards in both currencies was well described by a hyperbola-like function (Equation 3 ) isomorphic to that which describes temporal discounting.

\section{Method}

Subjects. Twenty-one students at the University of Warsaw participated as subjects.

Procedure. The procedure was similar to that in Experiment 1 except that the subjects chose between certain and probabilistic rewards (e.g. a " $10 \%$ chance of getting $\$ 100$ " and " $\$ 50$ for sure"). As in Experiment 1 , the U.S. dollar amounts were 100 and 1,000 . However, due to a change in the exchange rate, the amounts of the rewards in zlotys corresponding to the $\$ 100$ and $\$ 1,000$ rewards were 2.5 and 25 million Polish zlotys. Each of these amounts was presented at each of seven probabilities: $5 \%, 10 \%, 30 \%, 50 \%, 70 \%, 90 \%$, and $95 \%$ likelihood. There were 25 certain rewards ranging from $1 \%$ to $99 \%$ of the probabilistic amount. The order of presentation of conditions was counterbalanced in a manner analogous to that in Experiment 1 .

\section{Results and Discussion}

Although choices were presented in terms of probability (or percent likelihood), for purposes of analysis using Equation 3, the probability of a reward was converted to the odds against its receipt. As can be seen in Figure 3 , subjective value decreased systematically as a function of the odds against receipt of a probabilistic reward for both amounts in each currency. The curved lines represent Equation 3 fit to data from dollar rewards and the corresponding zloty reward, simultaneously using a single exponent parameter but separate discounting parameters (i.e., one value of $h$ was estimated for each currency) so that the discounting rates in the two currencies could be compared.

The upper left panel of Figure 3 shows group median data and best-fitting functions for the smaller amounts ( $\$ 100$ and 2.5 million $\mathrm{Zl}$ ); the upper right panel shows 


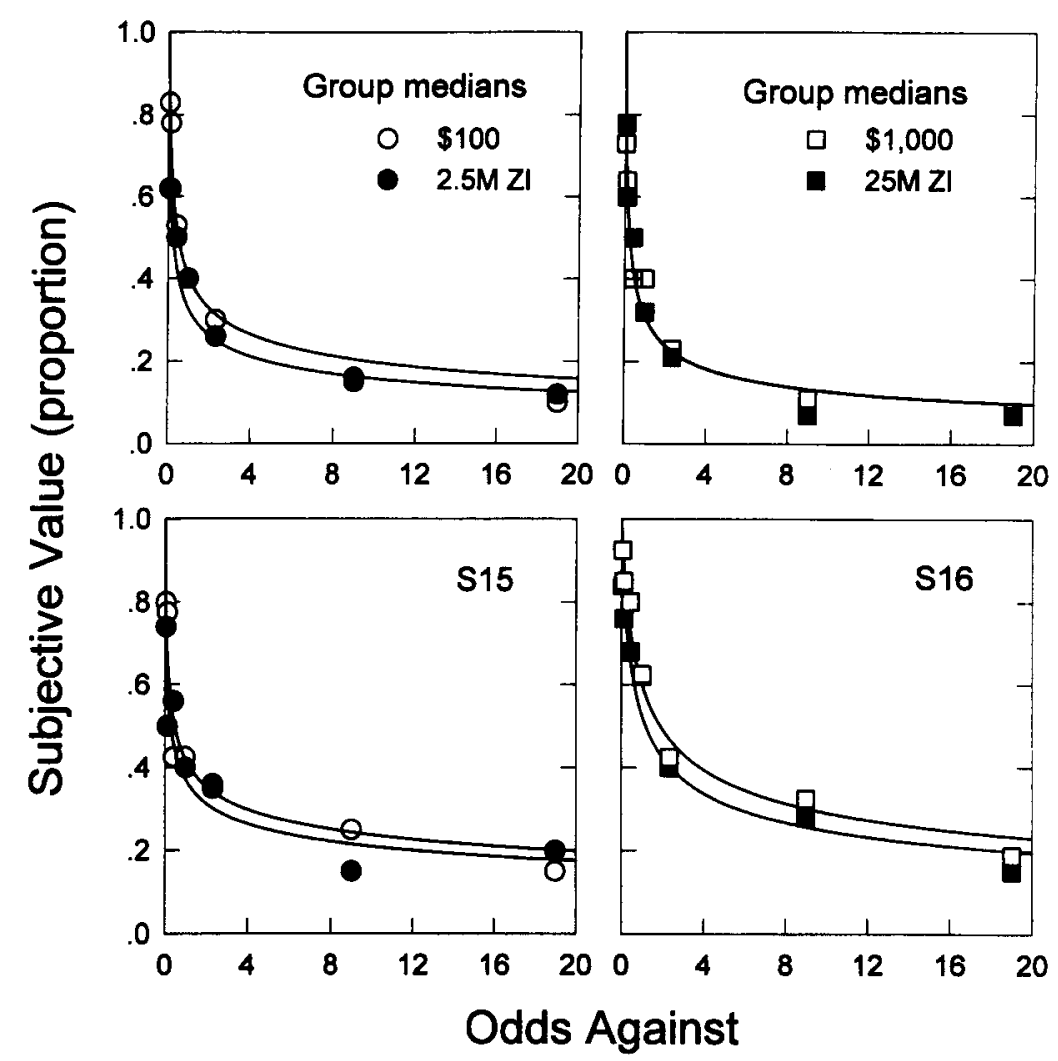

Figure 3. Subjective value of a reward as a function of the odds against its receipt. Data are the amount of certain reward equal in subjective value to the probabilistic reward (Experiment 2). The subjective value of a reward is represented as a proportion of its nominal amount. Curves represent the best-fitting hyperbola-like discounting functions (Equation 3). Top panels show group medians, and bottom panels show data from representative individuals.

the group data and best-fitting function for the larger amounts $(\$ 1,000$ and 25 million $\mathrm{Zl})$. A hyperbola-like function (Equation 3) accounted for $95.6 \%$ of the variance for the smaller amounts and $97.1 \%$ of the variance for the larger amounts. Equation 3 also described the data from individual subjects. Data and best-fitting functions for subjects with the median $R^{2} \mathrm{~s}$ for the smaller and larger amounts are presented in the two bottom panels. For these two subjects, Equation 3 accounted for $91.5 \%$ and $95.7 \%$ of the variance.

The exponent in Equation 3 fit to the group data was significantly less than 1.0 for both the small $(s=0.33)$ and large $(s=0.40)$ reward amounts $[t \mathrm{~s}(11)=17.60$ and 14.48 , respectively, both $p s<.001]$. This parallels the finding for the discounting of delayed rewards, where the exponent in Equation 1 was also significantly less than 1.0. These results are consistent with the view that the discounting functions for both delayed and probabilistic rewards are isomorphic (Rachlin et al., 1991). It should be noted that these results represent the first demonstration of the utility of incorporating an exponent in the hyperbolalike function describing the discounting of probabilistic rewards.
A major goal of Experiment 2 was to determine whether inflation produced specific or general effects. That is, the question was whether or not the steeper discounting of the more highly inflated Polish currency observed with delayed rewards would also be observed with probabilistic rewards. As can be seen in Figure 3, the discounting functions for comparable dollar and zloty rewards are quite similar. When Equation 3 was fit to the group median data shown in the upper panels, the values of the discounting parameters, $h$, did not differ significantly between the two currencies. For the $\$ 100$ and 2.5 million $\mathrm{Zl}$ rewards, the amount specified in zlotys was discounted more steeply, but not significantly so $[h \mathrm{~s}=4.74$ and 29.59 , respectively; $t(11)=1.88$ ]. For the $\$ 1,000$ and 25 million $\mathrm{Zl}$ rewards, the discounting rates were almost identical $[h \mathrm{~s}=18.12$ and 17.77 , respectively; $t(11)<1.0]$. These results stand in contrast to those of Experiment 1, where both large and small delayed rewards were discounted at significantly greater rates in the more rapidly depreciating Polish currency (compare Figure 3 with Figure 1).

Interestingly, there was no evidence of an amount effect of the kind seen with temporal discounting. Rather 
than smaller amounts being discounted more steeply, the discounting rate in the present experiment was equivalent to or even less steep for the smaller amounts. For the zloty rewards, the discounting parameter, $h$, did not differ significantly between larger and smaller amounts $[t(11)<1.0]$. For the dollar rewards, the larger amount was actually discounted more steeply than the smaller amount $[t(11)=3.15, p<.01]$.

\section{EXPERIMENT 3}

The results of Experiments 1 and 2 suggest that the effects of inflation on monetary decision making are specific to decisions involving future rewards. Future rewards specified in zlotys were discounted more steeply than future dollar rewards, but there was no difference in the discounting rates for probabilistic dollar and zloty rewards. Although inflation does not provide a complete explanation for the discounting of delayed rewards, the difference in the inflation rates in the two currencies appears to provide a possible explanation for the difference in the discounting of delayed zloty and dollar rewards and suggests that inflation modulates the rate of temporal discounting. The similarity in the rate at which probabilistic dollar and zloty rewards were discounted is consistent with this interpretation. Discounting of probabilistic rewards involves choices between outcomes that differ in certainty but in which rewards, if received at all, are received immediately. Because choices between certain and probabilistic rewards do not involve time, they were not affected by expectations concerning inflation rates.

A further test of this hypothesis was suggested by the introduction of the new zloty in 1995. In the years preceding 1994, there had been very high rates of inflation in Poland, averaging over 100\% between 1989 and 1993 (International Monetary Fund, 1996), and the zloty depreciated relative to the U.S. dollar. However, the new zloty was introduced during a period of decreased inflation, and the inflation rate at the time of Experiment 3 was the lowest in Poland in 8 years. This provided the opportunity to compare discounting of dollars with discounting of the new zlotys. If inflation was really the cause of the difference in the rate at which delayed rewards specified in dollars and old zlotys were discounted in Experiment 1, then, given the decreased rate of inflation in Poland in the 2 years preceding 1996 when Experiment 3 was conducted, the rates of temporal discounting should be more similar in the Polish and U.S. currencies. In addition, the introduction of new zlotys should have no effect on the discounting of probabilistic rewards, and equivalent discounting rates should be observed for probabilistic rewards in the two currencies.

\footnotetext{
Method

Subjects. Twenty-five students at the University of Warsaw (Poland) participated as subjects.

Procedure. The procedure was similar to that in Experiments 1 and 2 except that subjects chose both between immediate and delayed re-
}

wards and between certain and probabilistic rewards. Only one amount in each currency was used. These amounts were selected so that both the dollar and new zloty rewards were equivalent in worth according to the exchange rate at the time. In new Polish zlotys, the amount of the delayed and probabilistic rewards was 750; in U.S. dollars, the corresponding amount was 300 . Both of these amounts were presented at each of six delays ( 1 month, 6 months, 1 year, 3 years, 5 years, and 10 years) and at each of six probabilities $(5 \%, 10 \%, 40 \%, 70 \%, 90 \%$, and $95 \%$ likelihoods). There were 24 immediate or certain rewards, ranging from $1 \%$ to $99 \%$ of the delayed or probabilistic amount. Each subject participated in all four conditions (discounting of both delayed and probabilistic rewards, each specified in both dollars and new zlotys). The order of presentation of conditions was counterbalanced.

\section{Results and Discussion}

Figure 4 depicts the effects on the subjective value of increases in time until receipt of a delayed reward (left panels) and increases in the odds against receipt of a probabilistic reward (right panels). The curved lines represent the appropriate discounting functions (Equation 1 for delayed rewards and Equation 3 for probabilistic rewards) fit to data from the dollar reward and the corresponding new zloty reward simultaneously using a single exponent parameter but separate discounting parameters so that the discounting rates in the two currencies could be compared.

The top left panel of Figure 4 shows group median data'and best-fitting functions for discounting of the delayed $\$ 300$ and 750 new zloty amounts. Equation 1 accounted for $95.7 \%$ of the variance. The exponent was significantly less than $1.0[s=0.60 ; t(9)=3.42, p<.05]$. In contrast to the results of Experiment 1 , there was no difference between Polish and American currencies in rate of temporal discounting $[k \mathrm{~s}=0.181$ and $0.216 ; t(9)<1]$.

The top right panel of Figure 4 shows group median data and best-fitting functions for discounting of the probabilistic $\$ 300$ and 750 new zloty amounts. Equation 3 accounted for $95.9 \%$ of the variance. The exponent was significantly less than $1.0[s=0.47 ; t(9)=6.93, p<.01]$. As with temporal discounting, there was no difference between Polish and American currencies in discounting rates $[h \mathrm{~s}=20.24$ and $19.44 ; t(9)<1]$.

The bottom four panels show the data and best-fitting discounting functions for 2 subjects (S9 and S24) whose $R^{2} \mathrm{~s}$ for discounting the delayed and probabilistic rewards were both close to the median values for fits of Equations 1 and 3, respectively, to individual data. As can be seen, Equations 1 and 3 provide good fits to the individual data (for delayed rewards, the $R^{2} \mathrm{~s}$ were.838 and.854; for probabilistic rewards, the $R^{2} \mathrm{~s}$ were.875 and.889) and there was very little difference between discounting of dollar and new zloty rewards. At both the group and individual level, these results replicate those of Experiments 1 and 2 in showing that the same form of mathematical function can describe the discounting of both delayed and probabilistic rewards.

The primary purpose of Experiment 3 was to verify that the difference in temporal discounting of dollar and zloty rewards observed in Experiment 1 was due to differences 


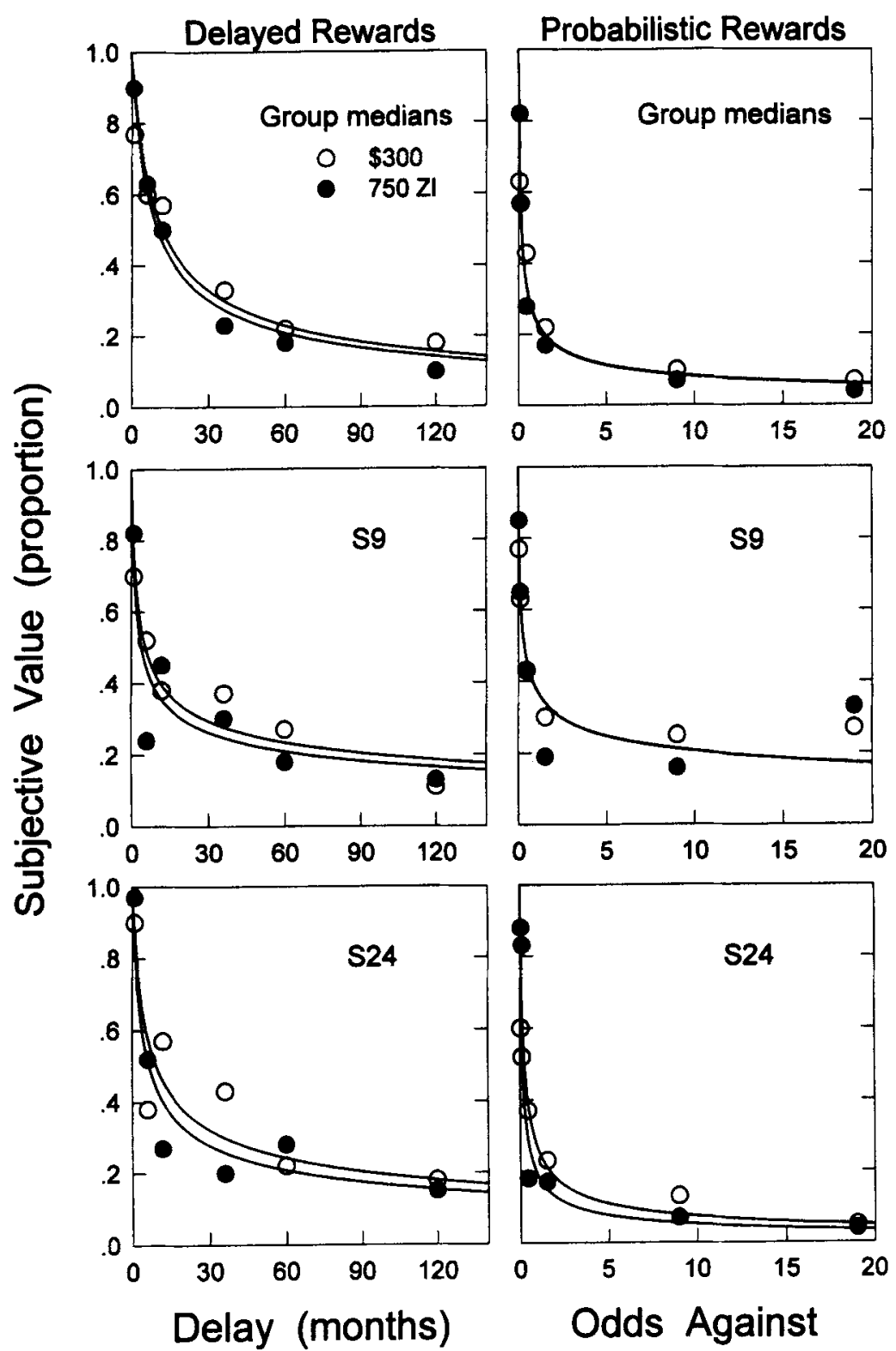

Figure 4. Subjective value of a reward as a function of the delay until its receipt (left panels) and the odds against its receipt (right panels). Data are from Experiment 3. Curves in the left and right panels represent the functions that best described the discounting of the delayed (Equation 1) and probabilistic (Equation 3) rewards, respectively. The top two panels show group medians; the bottom four panels show data from representative individuals.

in inflation and not to other differences between the two currencies. That is, if the highly inflated Polish currency around the time of Experiment 1 was the reason for the steeper temporal discounting of zloty rewards, steeper discounting would not be expected in Experiment 3, which was conducted during a period when the differences in inflation between the new Polish currency and the American currency were greatly reduced. Consistent with the hypothesis that the steeper temporal discounting of zloty rewards was tied to inflation rates, no differences in dis- counting were observed between the two currencies in Experiment 3.

\section{GENERAL DISCUSSION}

Taken together, the present results suggest that inflation has a specific effect on monetary decisions involving future rewards, and does not appear to affect decisions about probabilistic rewards involving immediate monetary outcomes. In the first experiment, conducted during a period preceded by high rates of inflation in Poland, future rewards specified in zlotys were discounted more steeply than future dollar re- 
wards. In contrast, the second experiment, conducted during the same period, showed no difference in the discounting rates for probabilistic dollar and zloty rewards. We assume that the difference in temporal discounting of zloty and dollar rewards was due to people's experience with inflation rates for the two currencies. Consistent with this interpretation, there was no difference in rates of discounting future zloty and dollar rewards in the third experiment, conducted during a period of lower inflation following introduction of the new zloty. The similar rates of discounting probabilistic rewards in the two currencies observed in Experiment 2 and again in Experiment 3 are also consistent with this interpretation. Such discounting involves choices between outcomes that differ in certainty but in which rewards, if received at all, are received immediately. Because probabilistic choices do not involve time, they were not affected by expectations concerning inflation rates.

Although it appears that people take inflation into account when making decisions about future rewards, this is not to say that they are actually making rational calculations on the basis of estimated inflation rates. The evidence against such mental calculations is clear. In the present study, as in previous experiments (Green, Myerson, \& McFadden, 1997; Kirby \& Maraković, 1996; Myerson \& Green, 1995; Raineri \& Rachlin, 1993), subjects discounted larger delayed amounts less steeply than they did smaller delayed amounts. In contrast, inflation affects all amounts equivalently and leads to equal proportional decreases in value. Moreover, inflation predicts an exponential decrease, and numerous studies have shown that the discounting function is not exponential in form (Green, Myerson, \& McFadden, 1997; Kirby, 1996; Kirby \& Maraković, 1995; Myerson \& Green, 1995; Rachlin et al., 1991).

Thus, the subjective value of future rewards differs from that expected on the basis of a rational model of inflation-sensitive decision making. Although inconsistent with such a model, the present results do demonstrate that people's choices regarding delayed and probabilistic monetary rewards are modulated by their experience with inflation in specific and roughly appropriate ways. That is, experience with inflation affects decisions regarding future rewards in direct relation to past currency-specific inflation rates and does not affect decisions regarding probabilistic, immediate rewards.

Importantly, the present analyses represent the first application of Equation 3 to the discounting of probabilistic rewards. Equation 3 provided accurate descriptions of the discounting of probabilistic rewards in both Experiment 2 and Experiment 3 at both the individual and group levels. Previous research has shown that a function of identical mathematical form (Equation 1) also accurately describes temporal discounting at both the individual and group levels (Green et al., 1994; Myerson \& Green, 1995), and this finding was replicated in Experiment 1 . The present results extend the generality of the hyperbola-like discounting function in two ways: first, by showing that such a function describes the discounting of both delayed and probabilistic rewards, and second, by showing that such a function describes both kinds of discounting in different currencies.

Thus, it appears that similar mathematical models can account for the discounting of both delayed and probabilistic rewards, suggesting that similar decision-making processes may be involved in both kinds of discounting. In fact, Rachlin and his colleagues (Rachlin et al., 1986; Rachlin et al., 1991) have proposed a single-process discounting model that explains discounting of probabilistic rewards in terms of an underlying temporal discounting process. They suggest that people evaluate probabilistic rewards in terms of the number of times, on average, one would have to gamble before winning. Because of the time that must elapse between gambles, the subjective value of a probabilistic reward decreases along with its probability due to the concomitant increase in the expected delay until its receipt. Myerson and Green (1995; Green \& Myerson, 1996) have also proposed that a single process may suffice to explain both kinds of discounting, but suggest that the single process is that underlying the discounting of probabilistic rewards. They suggest that people (and nonhuman animals) evaluate a future reward in terms of the risk that they will not actually receive it or will receive only part of it. Thus, the subjective value of both delayed and probabilistic rewards reflects a risk-assessment process. Although they differ in specifics, both models postulate a fundamental similarity between the discounting of delayed rewards and the discounting of probabilistic rewards. However, the fact that the same form of mathematical function (Equations 1 and 3) accurately describes changes in the subjective value of monetary rewards as delay or probability is varied by no means forces the acceptance of a single-process model. The present finding that inflation affects the discounting of delayed but not of probabilistic rewards poses problems for a simple single-process account, as does the finding that the two kinds of discounting are affected differently by variations in reward amounts. In the first experiment on temporal discounting, smaller amounts were discounted more steeply than larger amounts, whereas in the second experiment, the discounting rate was equivalent to or even less steep for the smaller amounts. Moreover, we have recently obtained further evidence that amount of monetary reward influences the discounting of delayed and probabilistic rewards in opposite ways in the same individuals (Green, Myerson, \& Ostaszewski, 1997).

The present findings show that inflation does not have a general effect on all aspects of decision making regarding rewards in a particular currency. Rather, the effects of inflation appear to be specific to certain kinds of decisions. Inflation influences decisions regarding future rewards, whereas decisions regarding probabilistic, immediate rewards are unaffected. Importantly, choices involving delayed and probabilistic rewards were both describable by a single mathematical model, suggesting that a unified account of both kinds of decision making was possible. Such an account must take into consideration the fact that inflation affects the discounting of delayed but not of probabilistic rewards, as well as the fact that amount may influence the discounting of delayed and probabilistic rewards in opposite ways. In spite of these differences, the fact that both forms of discounting have the same mathematical signature (i.e., Equations I and 3 are isomorphic) suggests that fundamentally similar, although not identical, processes are involved in decision making regarding delayed and probabilistic rewards.

In contrast to most previous behavioral economic research, which has focused on microeconomic issues, the present study examined the consequences of macroeconomic changes, specifically rates of inflation, for individual choice and decision making. We suspect that this unique aspect of the current approach may be profitably extended in various ways. Researchers might examine the effects of other macroeconomic phenomena, such as unemployment rate and the interest rates on loans and savings on diverse aspects of individual decision making, such as risk taking, health behaviors, and purchasing patterns. Such research might provide an empirical basis for predicting the effects of proposed macroeconomic interventions or for explicating the mechanisms underlying the observed consequences of past interventions.

Finally, although the microeconomic behavior of individuals and the macroeconomic behavior of aggregates represent different levels of analysis, these levels interact in a way that constitutes a feedback system. While much previous research in behavioral economics might be thought of as providing a better empirical basis for the path from the individual to aggregate behavior, the present study supplements such efforts by examining the other path in the loop (i.e., from the macroeconomic to the microeconomic). There is a need for a systems approach within behavioral economics that examines the ongoing interaction between individual choice and decision making and the behavior of economic aggregates, and theoretical analyses will need to be guided by empirical work on both paths.

\section{REFERENCES}

ALl.LSON, J. (1983). Behavioral economics. New York: Praeger

GaLlant, A. R. (1987). Nonlinear statistical models. New York: Wiley. Green, L., Fisher, E. B., Jr., Perlow, S., \& Sherman, L. (1981). Preference reversal and self-control: Choice as a function of reward amount and delay. Behaviour Analysis Letters, 1, 43-51.

Green, L., Fry, A. F., \& Myerson, J. (1994). Discounting of delayed rewards: A life-span comparison. Psychological Science, 5, 33-36.

GREEN, L., \& KaGEL, J. H. (EDS.) (1987). Advances in behavioral econamics (Vol. 1). Norwood, NJ: Ablex. 
Green, L., \& KaGel, J. H. (EDs.) (1990). Advances in behavioral economics (Vol. 2). Norwood, NJ: Ablex.

GreEN, L., \& Myerson, J. (1996). Exponential versus hyperbolic discounting of delayed outcomes: Risk and waiting time. American Zoologist, 36, 496-505.

Green, L., Myerson, J., \& McFadden, E. (1997). Rate of temporal discounting decreases with amount of reward. Memory \& Cognition, 25, 715-723.

Green, L., Myerson, J., \& Ostaszewski, P. (1997, May). Amount of reward has opposite effects on temporal and probability discounting. Poster presented at the annual meeting of the Association for Behavior Analysis, Chicago.

Hausman, J. A. (1979). Individual discount rates and the purchase and utilization of energy-using durables. Bell Journal of Economics, 10, 33-54.

HURSH, S. R. (1980). Economic concepts for the analysis of behavior. Journal of the Experimental Analysis of Behavior, 34, 219-238.

HURSH, S. R. (1984). Behavioral economics. Journal of the Experimental Analysis of Behavior, 42, 435-452.

INTERNATIONAL MONETARY FUND (1996). World economic outlook. Washington, DC: Author.

Kagel, J. H., Battalio, R. C., \& GreEn, L. (1995). Economic choice theory: An experimental analysis of animal behavior. Cambridge: Cambridge University Press.

KIRBY, K. N. (1996). Bidding on the future: Evidence against normative discounting of delayed rewards. Journal of Experimental Psychology: General, 126, 54-70.

KIRBY, K. N., \& MARAKOviĆ, N. N. (1995). Modeling myopic decisions: Evidence for hyperbolic delay-discounting within subjects and amounts. Organizational Behavior \& Human Decision Processes, 64, 22-30.

KIRBY, K. N., \& Maraković, N. N. (1996). Delay-discounting probabilistic rewards: Rates decrease as amounts increase. Psychonomic Bulletin \& Review, 3, 100-104.

LEA, S. E. G., TARPY, R. M., \& WEBLEY, P. (1987). The individual in the economy. Cambridge: Cambridge University Press

MAZUR, J. E. (1987). An adjusting procedure for studying delayed teinforcement. In M. L. Commons, J. E. Mazur, J. A. Nevin, \& H. Rachlin (Eds.), Quantitative analyses of behavior: Vol. 5. The effect of delay and of intervening events on reinforcement value (pp. 55-73). Hillsdale, NJ: Erlbaum.

Myerson, J., \& GREeN, L. (1995). Discounting of delayed rewards: Models of individual choice. Journal of the Experimental Analysis of Behavior, 64, 263-276.
Prelec, D., \& Loewenstein, G. (1991). Decision making over time and under uncertainty: A common approach. Management Science, 37, 770-786.

RACHLIN, H. (1989). Judgement, decision, and choice: A cognitive/ behavioral synthesis. New York: Freeman.

Rachlin, H., Logue, A. W., Gibbon, J., \& Frankel, M. (1986). Cognition and behavior in studies of choice. Psychological Review, 93, 33-45.

Rachlin, H., Raineri, A., \& Cross, D. (1991). Subjective probability and delay. Journal of the Experimental Analysis of Behavior, 55, 233244.

RAINERI, A., \& RaChLIN, H. (1993). The effect of temporal constraints on the value of money and other commodities. Journal of Behavioral Decision Making, 6, 77-79.

RoDriguez, M. L., \& Logue, A. W. (1988). Adjusting delay to reinforcement: Comparing choice in pigeons and humans. Journal of Experimental Psychology: Animal Behavior Processes, 14, 105-117.

STADDON, J. E. R. (ED.) (1980). Limits to action: The allocation of individual behavior. New York: Academic Press.

THALER, R. (1981). Some empirical evidence on dynamic inconsistency. Economics Letters, 8, 201-207.

\section{NOTE}

1. Curve-fitting was done using the same multiple nonlinear regression procedure for all three experiments in this study. The equation that was fit was $Y=1 /[1+(r+z * \Delta r) X]^{s}$, where $Y$ is the subjective value of the delayed or probabilistic reward and $X$ is delay or odds against. The discounting rate parameter (corresponding to $k$ and $h$ for delayed and probabilistic rewards, respectively) for one of the rewards being compared is represented by $r$, and the difference between the discounting rate parameters for the two rewards being compared is represented by $\Delta r$. The indicator variable, $z$, has the value 0 for one of the rewards and 1 for the other reward. Finally, the exponent $s$ represents the nonlinear scaling of amount or time. For example, when the smaller dollar and zloty rewards were compared, the discounting rate parameter for the dollar reward was $(r+0 * \Delta r)$ and the discounting rate parameter for the zloty reward was $(r+1 * \Delta r)$. Thus, the discounting rate parameters for the dollar and zloty rewards were $r$ and $(r+\Delta r)$, respectively. Statistical comparisons of discounting rates evaluated whether the value of $\Delta r$ estimated by the curve-fitting procedure differed significantly from zero (i.e., whether the two rewards were discounted at different rates).

(Manuscript received July 18, 1997; revision accepted for publication November 4,1997 .) 